\title{
Polyneuropathy and Balance
}

\author{
Kathrine Jáuregui-Renaud \\ Instituto Mexicano del Seguro Social \\ México
}

\section{Introduction}

Equilibrium or balance is a complex skill, which describes the dynamics of the control of posture in preventing falling. It is required for maintaining a position, remaining stable while moving from one position to another, as well as for performing activities and moving freely; it also serves to maintain bodily orientation to the environment. From a biomechanical perspective, balance can be defined as the ability to maintain or return the body's centre of gravity within the limits of stability that are determined by the base of support (i.e., the area of the feet) (Horak, 1987).

During upright stance, body movements and external perturbations apply continuous torques, if balance is to be maintained, those perturbations must be opposed by coordinated motor responses. To maintain balance, it is required to choose appropriate responses to each perturbation, to modify these responses on the basis of sensory input and, finally, to produce the needed muscular contractions (Era et al., 1996). Reflex movements regulate muscle force; whereas automatic reactions (stretch reflex-based movements) resist possible disturbances. Patients with polyneuropathy often have a deteriorated proprioception and/or motor function, which may imply deficits on postural control and gait, with an increased risk of falling.

\section{Postural control}

Postural control has been defined as the control of the body's position in space for the purposes of balance and orientation (Shumway-Cook \& Woollacott, 2000a), with variable attention requirements that depend on the task (Shumway-Cook \& Woollacott, 2000b; Teasdale \& Simoneau, 2001).

\subsection{Posture}

Postural control and its adaptation to the environment is based on background postural tone and on postural reactions (De Kleijn, 1923; Magnus, 1924), assisted by sensory feedback of mainly labyrinthine, visual, proprioceptive and cutaneous origin. Studies of normal stance have found the most rapid postural reactions to be a class of motor activities mediated primarily by inputs derived from the forces and motions of the feet upon the support surface (Nashner, 1977), foot sole and ankle muscle inputs contribute jointly to posture regulation (Kavounoudias et al., 2001). Locations of the centre of gravity close to the borders of limits of stability correspond to the region where balance cannot be maintained without moving the feet (Horak et al., 1989). 
A stance position that provides good stability is maintained mainly by ankle torque; when segmental oscillation is allowed to minimize the effect of perturbations, hip mechanisms are mainly used instead of ankle mechanisms (Figure 1). Postural tone is predominantly observed at the extensor muscles that counteract the effect of gravity and depends on the motor responses, particularly the integrity of the myotatic reflex loop, tonic labyrinthine reflexes and neck and lumbar reflexes. However, there are also supporting reactions and placing reactions (tactile, visual and vestibular), which adapt the activity of the postural muscles of the limbs to their function of body support.

Sensory information from multiple sources is crucial for the neural control of body orientation and body stabilization. Unsteadiness can be detected very early by proprioceptive sensory systems responding to motion and muscle stretch at the ankle, knee and trunk, as well as by head linear and angular accelerations sensed by the vestibular system (Allum et al., 1993), while visual inputs appear to primarily influence later stabilizing reactions to the initial balance corrections (Allum, 1985; Allum et al., 1993).
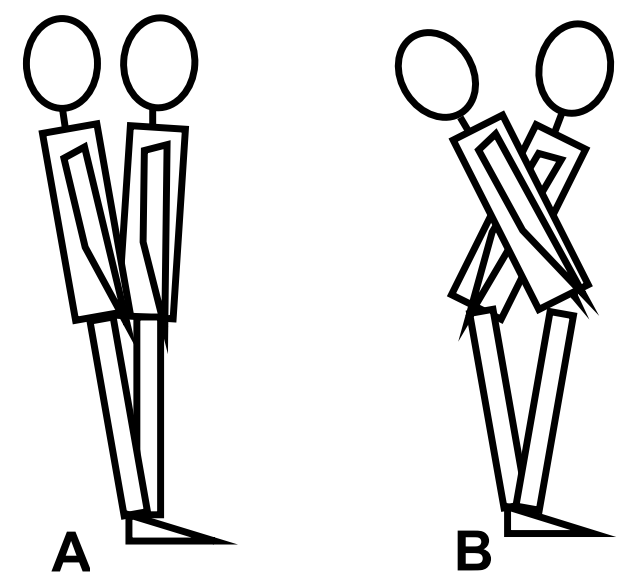

Fig. 1. Use of the ankle torque (A) or the hip (B), in order to maintain the centre of mass within the limits of stability.

The multiple sensory inputs may have an additive effect, without implying that the relative contributions of all sensory modalities have to be equivalent. Evidence support that the major contributor to balance control during quiet upright stance may be proprioceptive inputs (Allum et al., 1993; Horak et al., 1990). In the case of sensory conflict the nervous system may select one input that becomes dominant. However, a combined deficit of vestibular and somatosensory input may preclude adjustments to postural control (ArandaMoreno et al., 2009).

Since balance perturbations cause stretch responses at the ankle, knee, hip and neck joints, balance corrections imply the interaction among several proprioceptive inputs (Allum et al., 1993). Once balance corrections are triggered it is generally accepted that a confluence of proprioceptive and vestibular modulation to the basic centrally initiated template of activity establishes the amplitude pattern of the muscle response synergy (Diener et al., 1988; Forssberg \& Hirschfeld, 1994; Horak et al., 1994). Evidence suggests that somatosensory 
information from the legs may be utilized for both, direct sensory feedback and use of prior experience in scaling the magnitude of automatic postural responses (Inglis et al., 1994). Postural sway results in different but consistent patterns of discharge from muscle spindles, Golgi tendon organs and cutaneous receptors (Aniss et al., 1990). A number of different receptors in the legs and feet may provide the afferent information for the proprioceptive reflex but, because of the complex structure and dynamics of these receptors they don't transmit pure movement or torque information.

Disruption of somatosensory information from the feet only, by ischemic block at the ankle, results in normal muscle response latencies, but increased hip sway in response to support surface translations (Horak et al,. 1990). However, when the ischemic block is applied at the level of the thigh, which also blocks proprioceptive information from the muscles of the lower leg, body sway increases even more (Diener et al. 1984a).In standing humans, there is a notable attenuation of postural sway at frequencies above $1 \mathrm{~Hz}$; sway may appear at this frequency when afferents from the leg muscles and feet are blocked with ischemia (Diener et al., 1984a; Mauritz \& Dietz, 1980); proprioceptive reflex response to sway may also contribute significantly to stability at these frequencies (Fitzpatrick et al., 1992; Oppenheim et al., 1999). Also, significant attenuation is due to low-pass filtering by the body's inertia and the short-range visco-elastic stiffness of the ankles and leg muscles (Allum \& Buidingen, 1979; Nashner, 1976).

The attention requirements for postural control vary depending on the postural task (Teasdale \& Simoneau, 2001), the age of the individual and their balance abilities (Camicioli, 1997; Kerr, 1985; Woollacott, 2002). Even in young adults, postural control is demanding of attention; however not all cognitive tasks affect postural control in the same way, balance tasks may disrupt spatial but not nonspatial memory performance (Kerr et al., 1985). In both healthy and older adults with balance impairment, studies using dual task paradigms to examine attention requirements of balance control when performing a secondary task suggest that these are important contributions to instability, depending not only on the complexity of the task, but also on the type of the second task being performed (Camicioli et al., 1997; Woollacott \& Shumway-Cook, 2002).

\subsection{Gait}

Human walking requires neural and muscular coordinated actions with adequate skeletal function; the generation and regulation of walking involve all levels of neural substrates, as well as an adequate function of several effectors. Walking differs from standing balance in that the centre of mass constantly moves beyond the base of support, and in fact the support leg can do little to alter this motion (Winter \& Scott, 1991).

The gait cycle is divided into stance and swing phases:

STANCE PHASE:

- initial contact,

- loading response,

- mid stance,

- terminal stance,

- pre-swing.

SWING PHASE:

- initial,

- mid,

- terminal swing. 
During initiation and termination of gait, ankle and hip mechanisms control the trajectory of the centre of pressure to ensure the desired acceleration and deceleration of the centre of mass; shifts of the centre of pressure are controlled by the hip abductors/adductors and the ankle plantar dorsi-flexors (Winter, 1995).

The control of placement of the swing foot can be used to stabilize balance by manipulating the redirection of the centre of mass that occurs as the foot contacts the ground (Townsend, 1985) (Figure 2). Besides peripheral inputs and proprioceptive reflexes processed in the spinal cord, the cerebellum, basal ganglia, and cortical mechanisms contribute to the motor control necessary for normal gait (Dietz, 2001).

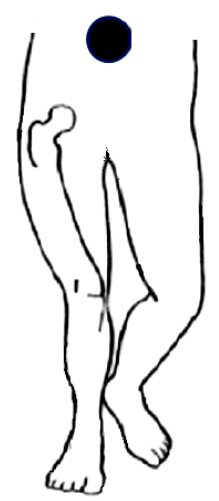

Fig. 2. Position of the center of mass.

\section{Evaluation of postural control}

\subsection{Clinical history}

Effective assessment of balance requires an understanding of the many systems underlying postural control. To assess a history of instability or walking difficulty, the clinician should evaluate the affected movements and circumstances in which they occur, as well as any other associated symptoms about postural control, motor control, muscular force, sensory deficiency and balance, including limitations imposed by pain, by restriction of joint range of motion and by muscle strength.

A history of falls is common in patients with disorders of balance and gait. If the occurrence of fall(s) is identified, it is important to investigate the circumstances in which each fall occurred, such as the environment (eg. environmental light) and the specific situation at the moment of falling (eg. activity \& movement). Spontaneous falls are frequent in patients with sensory deficit (eg. sensory ataxia), who usually report unsteadiness that may be exacerbated under circumstances in which the visual information is reduced (eg. while walking in the dark).

\subsection{Balance examination}

In order to evaluate balance, a neurological examination is required. A proper evaluation should contain an examination of motor and sensory function, including: spinal column, joints with their range of motion, muscle tone and strength, reflex responses, proprioception, primary sensation as well as higher order aspects of sensation. Additional care should be taken to observe the postural control and gait, and to identify vestibular disorders. 
The postural control during upright stance has to be observed, including the width of the stance, the symmetry/balance at the level of the joints and the trunk posture, during at least the following conditions: with the eyes open, with the eyes closed, while standing with the feet together, while balancing on the two legs and while standing on foam. Additionally, the reaction to push gently the patient while standing will provide information on postural reflexes. Patients with a sensory deficit usually show a wide base of support while standing, and they also have difficulties on maintaining balance when closing their eyes. This finding can easily be observed during Romberg's test.

To evaluate gait, a sensory-motor evaluation should be performed, as well as a postural and skeletal examination (Thompson \& Marsden, 1996). While observational gait assessment is relatively subjective in nature, instrumented gait assessment is restricted by the fact that it is laboratory based (Toro et al., 2003).

During observation of walking it is important to analyse the initiation, stepping and the associated movements. The initiation of walking requires a complex interaction of postural changes which could be affected by several factors; during stepping, at least the speed of walking, the rhythm and the length of each stride have to be evaluated; the synergy of the associated mobility of the trunk, arms and head can be helpful to identify a problem. In patients with somatosensory deficit the patient may guide the movement by vision and hit the floor with the heel.

A neuro-otological evaluation may detect vestibular deficiencies, which could be compounding polyneuropathy (eg. diabetic patients) or may have a different origin (central or peripheral). A detailed evaluation of long tracts, cranial nerves, motor control, the eyes and the ears should be performed. The evaluation of eye movements may include (Leigh \& Zee, 1991): the inspection of the eyes as the patient keeps the head stationary and fixes upon a distant point to observe spontaneous eye movements (eg. spontaneous nystagmus), the range of movements in the plane of each pair of extra-ocular muscles, the eye convergence, the fast eye movements guided by visual stimuli (saccades), the slow eye movements guided by visual stimuli (smooth pursuit), the opto-kinetic response, the eye movement response to passive head movement and the eye movements during and after postural changes.

When spontaneous nystagmus is observed, it is classified according to the position of the eyes in which it is observed (Figure 3) and according to the direction of the fast phase: horizontal (right/left), vertical (up/down) or torsional (clockwise/ counter-clockwise).

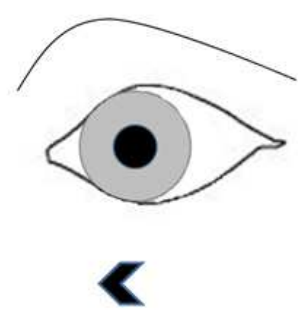

1st DEGREE

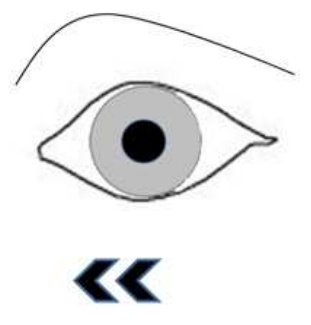

2nd DEGREE

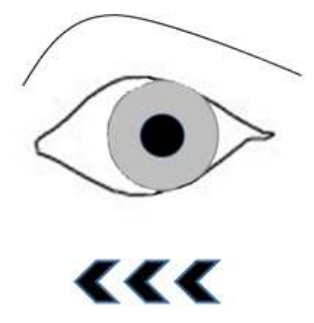

3rd DEGREE

Fig. 3. Classification of horizontal spontaneous nystagmus: 1st degree when present only with gaze deviation in the fast phase direction, 2nd degree when present in primary gaze, and 3rd when it is evident even during gaze deviation in the direction opposite to the fast phase direction. 
When it is not possible to identify slow and fast phases, it is a pendular nystagmus of central origin. To evaluate small eye movements or prevent visual fixation, the nystagmus can be observed during observation of the retina by ophthalmoscopy. However, in interpreting the findings during ophthalmoscopic examination, it should be noted that because the retina is behind the centre of rotation of the eye, the direction of the eye movement is the opposite of that observed by viewing the front of the eyes. When postural changes, such as the Hallpike manoeuvre, elicit postural nystagmus, the characteristics of its presentation suggest a peripheral or a central origin: a peripheral vestibular origin is consistent with a latent period, adaptation and fatigability, with accompanying vertigo.

To evaluate the vestibular system and its relationships with other sensory inputs and the oculo-motor system, specific tests have to be performed, including eye movement recordings and vestibular reflexes. There are several tests that require high technology equipment. However, the simplest test that can be applied with minimal technology is the bi-thermal caloric testing at $30^{\circ} \mathrm{C}$ and $44^{\circ} \mathrm{C}$, to analyse the oculo-motor response to labyrinth stimulation by thermic changes.

When measuring balance, different aspects can be considered: neurophysiological recordings of the electric potentials due to muscle activation, kinematics concerned with movement itself and kinetics, which is concerned with the forces and the moments of forces that are developed during movements. Posturography platforms are used to record kinetic data. The analysis of the centre of pressure is used to assess the ability of a subject to maintain a stable stance over a period of time, while standing on the force platform (wearing a safety harness). Although it is difficult to unravel the vestibular, visual and somatosensory influence on balance, posturography platforms allow the general evaluation of the interactions between vestibulo-spinal and proprioceptive reflexes for balance control, and the influence of vision. The simplest method to record postural sway uses a force plate. These devices measure the position of the centre of force, according to the feet pressure on the surface of support, which is a good estimate of the position of the centre of mass during upright stance. Unfortunately, static force plates do not yield controlled stimulus-response measures.

To overcome the limitations of static force platforms, several moving force platforms have been designed. These devices can maintain a constant angle between the foot and lower leg and the movement of the visual enclosure of the platform can be coupled to the body sway.

Dynamic posturography is useful in the assessment of the equilibrium performance in patients with polyneuropathy. It has been proposed as a tool for the follow-up, to follow course of the balance deficit related to the disorder (Jáuregui-Renaud et al., 1998; Vrethem et al., 1991). In short, dynamic posturography uses a computer-controlled movable platform and movable visual surrounding; the platform and visual surrounding are either stable or moving, referenced to the patient, creating a disturbed proprioceptive and visual input (Nashner \& Peters, 1990) (Figure 4). The test protocols usually include a sensory organization protocol, a motor control protocol and adaptation protocols.

Gait analysis includes the measurement of joint kinematics (concerned with movement itself), and kinetics (concerned with the forces developed during movements); other measurements commonly made are electromyography, oxygen consumption and foot pressures. Neurophysiological recordings are used to analyse the involvement of specific muscles or muscle groups during movement and the latency of their activation; a recording is made of the generation and propagation of action potentials in nerve and muscle cells. 
Kinematics can be evaluated by opto-electric methods, among them is the use of optoelectric imaging systems to record the movements of body segments and their directions; another option is to track the position of the body segments by measuring the positions of light-emitting markers.

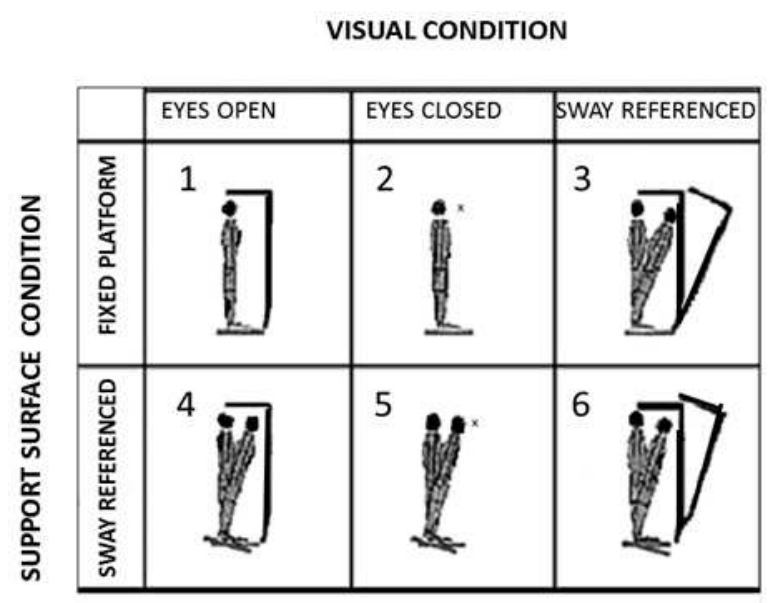

Fig. 4. Sensory conditions of Dynamic Posturography (Nashner \& Peters, 1990): 1. Eyes open with fixed surrounding and fixed support. 2. Eyes closed with fixed support. 3. Swayreferenced surrounding with fixed support. 4. Eyes open with fixed surrounding and swayreferenced support. 5. Eyes closed with sway-referenced support. 6. Sway-referenced surrounding with sway-referenced support.

Research investigating human gait has traditionally focused on kinematic variables (eg, joint angles, stride length, walking velocity). Power is the kinetic variable that reflects the rate of work performed at a given joint (Mueller et al., 1994). It can be calculated by taking the product of the moment and the joint angular power; it will be positive when the moment and the angular velocity have the same direction. A positive power usually indicates that the muscle is generating mechanical energy (concentric contraction).A negative power usually indicates that the muscle is absorbing mechanical energy (eccentric contraction) Although muscle power varies during walking, the power generated by the plantar flexors at push-off is typically the largest power burst recorded during the gait cycle (Minor et al., 1994).

Other methods to evaluate balance include standardized scales and clinical tests. Functionally directed balance tests are typically dynamic tests that measure a person's ability to maintain balance as the subject walks or performs tasks to simulate the actions of daily activities and work. The choice of a clinical tool needs to reflect the purpose for which the tool is to be applied. If the purpose is to screen for high-risk populations, a tool is needed that is quick and easy to apply, yet has good sensitivity and specificity. If the purpose is to reduce risk, the tool needs to reliably identify remediable risk factors on which interventions can be focused.

Among the functional balance scores and performance scales are the "Get up and go test" (Podsiadlo \& Richardson, 1991), the Berg's Balance Scale (1992) and the Tinetti scale (1986). 
Although these scores and scales do not give specific information about the origin of balance impairment, they are useful to evaluate and follow-up deficits that are evident on circumstances of daily life. There are also a number of fall-risk assessment tools available with some evidence to support their use in predicting risk of falls (Scott et al., 2007).

On the basis of predictive values of $70 \%$ or higher for both sensitivity and specificity of the instrument (Olivier et al., 2004; Perell et al., 2001), some tools tested with predictive validity are the $5 \mathrm{~min}$ walk, the five-step test and the Functional Reach (Wang et al., 2005), the Mobility Fall Chart (Lundin-Olsson et al., 2000) and the evidence based risk assessment tool (Olivier et al., 1997).

To interpret the results of any balance evaluation, several factors have to be considered. Regardless of the technique of measurement used, standing body sway has been generally shown to increase with age, with an increased dependence on vision (Colledge et al., 1994; Poulain \& Giraudet, 2008). Deterioration in balance function clearly starts at relatively young ages and further accelerates from about 60 years upwards; males tend to have more pronounced sway than females, and the difference increases in the older age groups (Era et al., 2006). Evidence suggests that body weight may also have an influence on postural stability, particularly when vision is not available (Cruz-Gómez et al., 2011).

Among other factors, in patients with polyneuropathy, adaptive compensation to changes in biomechanical factors and vice versa may complicate evaluation of the source of balance impairment. Plantar ulceration is the most common consequence of the loss of sensation in the lower extremities, patients with diabetes may have limited joint mobility of the feet independent from peripheral neuropathy (Shinabarger, 1987), as well as visual deficiency related to retinopathy.

\section{Postural control and gait of patients with polyneuropathy}

Intact balance is required to maintain postural stability as well to assure safe mobility during activities of daily life. Maintaining balance encompasses the acts of maintaining, achieving or restoring the body center of mass relative to the limits of stability, which are given by the base of support (Pollock, 2000). Reduced somatosensory information from the lower limbs in human beings alters the ability both, to trigger postural responses to perturbations in stance and to scale the magnitude of the response to the magnitude of the perturbation (Diener et al., 1984b; Horak et al.,1990; Jáuregui-Renaud et al., 1998) (Figure 5). Performance on clinical balance tests is associated with electrophysiological and clinical measures of neuropathy (Goldberg et al., 2008).

Subjects with severe neuropathy are significantly less stable than normal subjects and patients without neuropathy (Oppenheim et al., 1999). Patients with polyneuropathy exhibit decreased stability while standing and walking (Boucher et al., 1995; Richardson et al., 1992; Simoneau et al., 1994), as well as when subjected to dynamic balance conditions (Bloem et al., 2000; Inglis et al., 1994). Evidence suggests that the role of peripheral sensory information in postural control may differ depending both, on the postural control task and on the quality of the sensory information available (Inglis et al., 1994). Compared to healthy age/sex matched controls, in patients with severe peripheral neuropathy without known origin, visual and vestibular input cannot fully compensate for the impairment in proprioception, with progressive deterioration of balance (Jáuregui-Renaud et al., 1998) (Figure 6). 


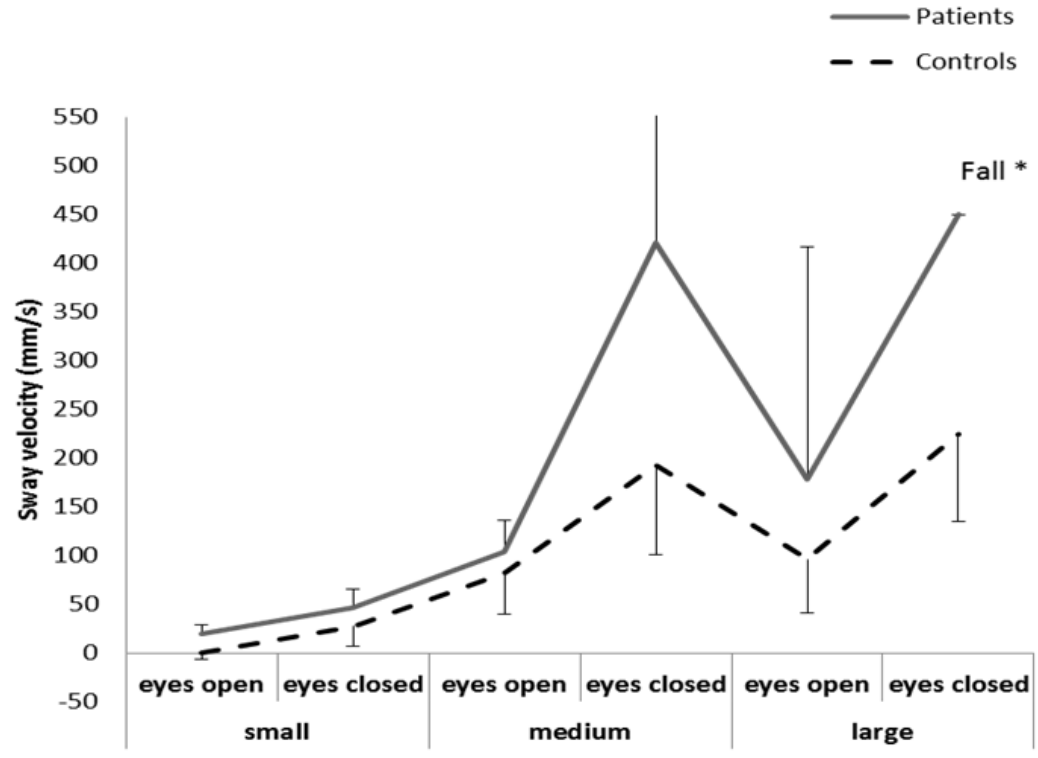

Fig. 5. Mean and standard deviation of the mean of sway velocity of 14 patients with polyneuropathy of unknown origin and 14 age/sex matched healthy controls, during responses to small, medium and large anterior-posterior translations of the support surface fashion (Jáuregui-Renaud 1998). $\left(^{*}\right.$ ) During the large translations with eyes closed 13/14 patients fell.

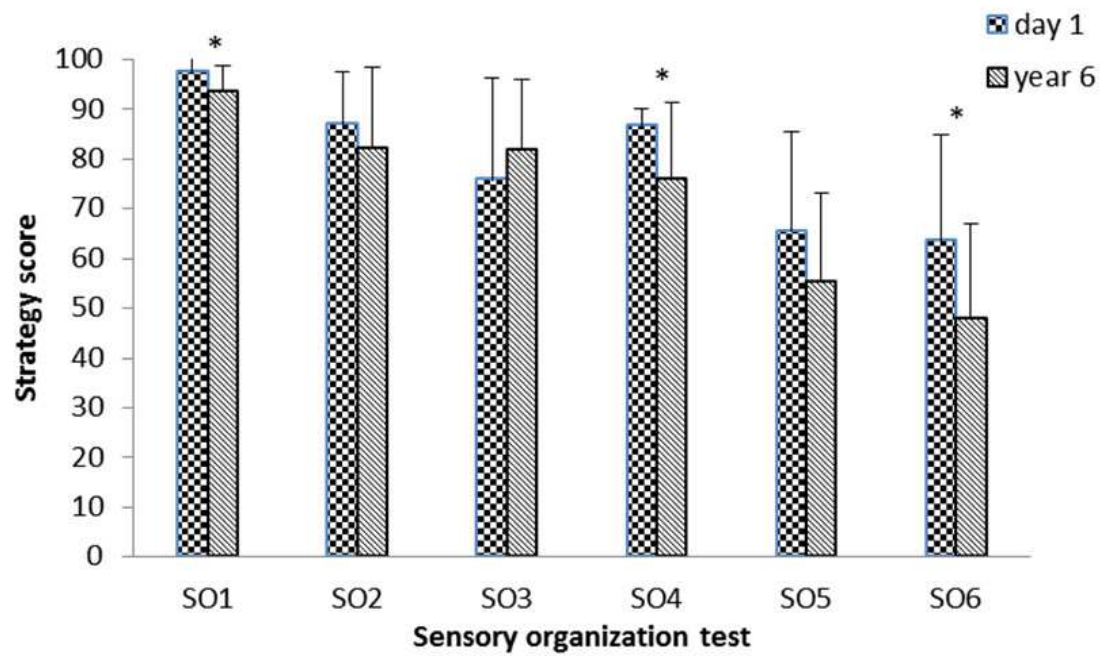

Fig. 6. Mean and standard deviation of the mean of the strategy scores, related to the amount of shear forces generated, during the six sensory organization conditions of dynamic posturography, of 14 patients with polyneuropathy of unknown origin during a 6 year follow-up (Jáuregui-Renaud 1998). ( $\left.{ }^{*} \mathrm{p}<0.05\right)$ 
A frequent source of polyneuropathy is diabetes mellitus; its clinical characteristics make it a unique neurophysiological model to study balance problems related to polyneuropathy. Peripheral diabetic neuropathy is initially characterized by a reduction in somesthesic sensitivity due to the sensitive nerve damage.

The prevalence of sensory ataxia in diabetic patients ranges from $10 \%$ to $90 \%$ depending on screening protocol and criteria to define neuropathy (Vinik et al., 2000). With the progression of the diabetic neuropathy, motor nerves are damaged. In neuropathic patients, the peroneal nerve and the sural nerve frequently present abnormalities in electrophysiological tests (Dyck et al., 1985). However, the frequency of balance symptoms in diabetic patients is related to both the time elapsed since the diabetes was diagnosed and the history of peripheral neuropathy and retinopathy (Jáuregui-Renaud et al., 2009), and the bio-mechanical impairment caused by progression of foot complications has to be considered in addition to the instability caused by neuropathy (Kanade et al., 2008).

During posturography, compared to patients without neuropathy, patients with diabetic polyneuropathty may show: larger area of sway and speed of sway (Boucher et al., 1995; Uccioli et al., 1995), as well as larger center of pressure range (Simoneau et al., 1994) and increased oscillation at 0.5-1 Hz (Oppenheim et al., 1999). In this group of patients, the standing balance area of sway may predict functional gait (Manor \& Li, 2009).

Although patients with diabetic sensory neuropathy may show a normal distal-to-proximal dorsal muscle activation pattern in response to backward support surface translation, their electromyography onset latencies may be delayed by $20-30 \mathrm{~ms}$ at all segments when compared with age-matched control subjects (Meyer et al., 2004). The consistency in delays at all segments, despite the fact that diabetic neuropathy is more severe in distal limb segments than in proximal leg segments and the trunk, suggests that slowed sensory conduction in the lower legs results in the late onset of an otherwise intact, centrally programmed response (Meyer et al., 2004).

The most effective gait allows a person to move over a variety of terrains safely and with minimal effort. In neuropathic gait, there is a longer support phase related to a flexor dysfunction of the tibialis anterior during the flat foot phase and also to a typical delay of the recruitment of tibialis anterior, peroneus brevis and longus and soleus (Mueller et al., 1994; Abboud et al., 2000). Polyneuropathy modifies the amount and the quality of the information necessary to motor control. Consequently, there is an increase in instability during gait (Richardson et al., 1992). The gait velocity, step length, amplitude of ankle movement, ankle moments of force, power and anterior-posterior ground reaction force variables are smaller in diabetic patients with polyneuropathy, compared to control subjects (Mueller et al. 1994). This group of patients may show decreased ankle plantar-flexor strength and ankle mobility compared with age-matched controls, as well as increased frontal plane motion at the ankle (Katoulis et al., 1997). The use of dual force platforms has also shown that ankle evertor/ invertor motor activities are impaired (Lafond et al., 2004). These deficits appear to contribute to the limited ankle motion, ankle moments, ankle power, stride length, and velocity during walking. Also, reaction times while walking may be higher for diabetic neuropathic patients than for control subjects (Courtemanche et al., 1996).

Diabetic patients with polyneuropathy may show greater hip moments and power than ankle moments and power during terminal stance, opposite to the pattern shown by control subjects (Minor et al., 1994); they appear to pull their leg forward using hip flexor muscles rather than pushing the leg forward using plantar flexor muscles. 
During gait, the motor system will generate motor responses according to the mechanical loads received by the foot in order to attenuate the load in this initial contact. In diabetic patients with polyneuropathy, the study of the plantar pressure distribution and electromyographic activity of tibialis anterior, peroneus brevis, peroneus longus and soleus muscles during gait, have shown that all these muscles present an important delay in their recruitment (Abboud et al., 2000). The feet of the diabetic patients contact the ground in midstance earlier than what has been verified for healthy subjects, and the forefoot keeps longer contact with the ground during stance phase and presents higher peak pressure in comparison with other plantar areas (Abboud et al., 2000).

The tibialis anterior has a fundamental role in the initial contact phase of gait. Its function is to reduce the initial shock of the forefoot during the flat foot phase (Winter \& Scott, 1991). In patients with polyneuropathy, if a delay in the tibialis anterior activation occurs, the shock attenuation mechanism will fail, and higher loads on the forefoot are generated, with an alteration in the contribution of the ankle (Sacco \& Amadio, 2003).

Older women with polyneuropathy may walk reasonably stable on flat surfaces with good lighting, but their gait speed under these ideal circumstances is somewhat slower than needed in the community for crossing streets; under ideal conditions they are able to regulate their gait to a similar degree as healthy women of similar age, but at the cost of speed and efficiency. In addition, when the environment is challenging, the gait speed of this group of patients may be severely reduced (Richardson et al., 2004).

\section{Polyneuropathy and falls}

A fall is often defined as inadvertently coming to rest on the ground, floor or other lower level, excluding intentional change in position to rest in furniture, wall or other objects (World Health Organization, 2007). Falls occur as a result of complex interactions among demographic, physical and behavioural risk factors. Risk factors have been identified and categorized as intrinsic or extrinsic factors: intrinsic factors include demographic and biological factors, while extrinsic factors encompass environmental and behavioural factors (WHO, 2007).

Gait and balance problems are the most frequently cited cause of falling in all age and gender groups, but women have a higher percentage of falls than men (Talbot et al., 2005). In older people, falls are a risk factor for disability and may account for $40 \%$ of all injury deaths (Rubinstein, 2006). While approximately one in three older people fall each year, this proportion varies depending on the country and the target population studied (WHO, 2007). In this age group, the majority of falls occur while walking on a level surface during ordinary daily activities in the absence of hazardous behaviour (Talbot et al., 2005; Niino et al, 2000).

During physical examination of older patients, peripheral neurologic deficits are commonly found. Among the causes are: diabetes mellitus, alcoholism, nutritional deficiencies, malignancies, and autoimmune diseases. When somato-sensation is affected (eg. neuropathy or after anaesthesia) a decline in control of balance may occur, associated with an increased risk of falling (Simoneau et al., 1994; Van Deursen \& Simoneau 1999). However, quantifying the somatosensory loss cannot fully predict falls. Evidence support that older persons with polyneuropathy are at a markedly increased risk for injurious falls (Cavanah et al., 1992; Richardson et al., 1992). 
Epidemiological surveys have established that a reduction of leg proprioception is a risk factor for falls in the elderly (Lord et al., 1996; Richardson \& Hurvitz., 1995). Even with vision, the postural stability of neuropathic patients is impaired and may put them at higher risk of falling when performing challenging daily tasks (Boucher et al., 1995).

A study focused to investigate if polyneuropathy could be just a marker for a comorbidity (e.g., central nervous system dysfunction) as the true cause of falls, have shown that polyneuropathy is a true risk factor for falls in the elderly (Richardson \& Hurvitz 2005). In this group of patients, loss of plantar sensation may be an important contributor to the dynamic balance deficits and increased risk of falls (Meyer et al., 2004). Cavanagh et al. (1992) reported that patients with diabetes and peripheral neuropathy were 15 times more likely to report an injury (fall, fracture, sprained ankle, or cuts and bruises) during walking or standing, compared to subjects in a control group of patients with diabetes but no peripheral neuropathy.

The ability to re-weight sensory information depending on the sensory context is important for maintaining stability when an individual moves from one sensory context to another, such as a flat walking surface to an uneven surface or a well-lit sidewalk to a dimly lit garden. Individuals with peripheral vestibular loss or somatosensory loss from neuropathy are limited in their ability to re-weight postural sensory dependence (Jáuregui-Renaud et al., 1998; Agrawal et al., 2010) and thus, they are at risk of falling in particular sensory contexts. Older patients with peripheral neuropathy have a high rate of falls associated with walking on irregular surfaces (DeMott et al., 2007). Compared to healthy subjects, patients with diabetes and peripheral neuropathy may show poorer balance during standing in diminished light compared to full light and no light conditions (Petrofsky et al., 2006).

However, peripheral neuropathy and retinopathy may not completely account for the measures of physical performance significantly associated with increased risk for falling (Shwartz et al, 2008). Subjects with lower extremity weakness, usually measured by knee extension, ankle dorsiflexion, and chair stands, have a 1.8-fold increased risk of falling and three-fold risk for recurrent falls (Moreland et al., 2004). Among other risk factors, the occurrence of falls may be significantly associated with a best-corrected Snellen visual acuity of less than 6/12 (Kuang et al, 2008), the use of four or more prescription medications (Tinetti et al., 2003; Huang et al., 2009) and foot deformities (Cavanah et al., 1992; WHO, 2007). In addition, fear of falling and poor physical performance commonly occurs after falls (Tinetti et al., 1994). One of the major consequences of fear of falling is the restriction and avoidance of activities, which results in muscle atrophy and loss of strength, especially in the lower extremities, and may also be predictive for future falls (Maki et al., 1991; Delbaere et al., 2004).

In order to modify the risk of falling a multidisciplinary approach is required; some of the modifiable or preventable risk factors may include: balance and gait deficits, psychological factors like fear of falling, polypharmacy, lower extremity impairments and environmental threats. However, crucial to the success of any intervention is changing the beliefs, attitudes and behaviour of both, the people/community and the health/ social care professionals.

The World Health Organization Model for Prevention of Falls is built around three pillars (WHO, 2007):

1. building awareness of the importance of falls prevention and treatment;

2. improving the assessment of individual, environmental and societal factors that increase the likelihood of falls; and

3. facilitating the design and implementation of culturally-appropriated evidence based interventions that will significantly reduce the number of falls. 
Health professionals, particularly those who take care of patients with polyneuropathy, have a critical opportunity to identify individual risk factors for falling and to identify the best evidence to select the most appropriate evaluations and interventions needed for the prevention and treatment of falls.

\section{Conclusions}

Balance encompasses the acts of maintaining, achieving and restoring the body centre of mass relative to the limits of stability, in preventing falling.

Postural stability is dependent upon integration of signals from the somatosensory, visual and vestibular systems, with central integration, to generate motor responses.

Assessment of balance requires an understanding of the many systems underlying postural control. To evaluate balance, a neurological examination is required, including motor and sensory function, as well as postural control and gait. Measures of standing balance may be performed using neurophysiological recordings of the electric potentials due to muscle activation, kinematics concerned with movement itself and kinetics.

The analysis of the centre of pressure during upright stance is used to assess the ability of a subject to maintain a stable stance over a period of time. The simplest method to record postural sway uses a force plate to measure the position of the centre of force, according to the feet pressure on the surface of support (Static posturography). To further evaluate the control of posture, moving force platforms and visual surroundings have been designed to modify the somatosensory and visual inputs (Dynamic posturography). Gait analysis may include the measurement of joint kinematics and kinetics, electromyography, oxygen consumption and foot pressures Other methods to evaluate dynamic balance include standardized scales, which are usually directed to measure a person's ability to maintain balance while performing tasks to simulate the actions of daily activities.

Polyneuropathy modify the amount and the quality of the sensorial information that is necessary for motor control, with increased instability during both, upright stance and gait. Compared to healthy controls, in patients with severe peripheral neuropathy, visual and vestibular input may not fully compensate for the impairment in proprioception, with progressive deterioration of balance. During posturography, patients with diabetic polyneuropathty may show an increase of both the area of sway and the speed of sway, as well as an increased sway at medium - high frequencies. In this group of patients, gait may also be deficient, even under ideal circumstances (level surface with good lighting).

Spontaneous falls are frequent in patients with sensory deficit, who usually report unsteadiness that may be exacerbated under circumstances in which the visual information is reduced. In patients with peripheral polyneuropathy, reduced somatosensory information from the lower limbs also deteriorate the ability to produce adequate postural responses to perturbations in stance.

Gait and balance problems are the most frequently cited cause of falling in all age and gender groups, and polyneuropathy is a risk factor for falls in the elderly. Individuals with somatosensory loss from neuropathy are limited in their ability to re-weight postural sensory inputs and, they are at risk of falling particularly in challenging contexts. However, several factors may account for the measures of physical performance significantly associated with increased risk for falling, such as foot deformities, deficient visual acuity, polypharmacy and fear of falling. 
The health care providers, who take care of patients with polyneuropathy, have a critical role to play in identifying individual risk factors for falling and selecting appropriate interventions for the prevention and treatment of falls.

\section{References}

Abboud, R.J., Rowley, D.I., Newton, R.W. (2000). Lower limb muscle dysfunction may contribute to foot ulceration in diabetic patients. Clinical Biomechanics (Bristol, Avon), Vol. 15,No. 1, (January 2000), pp. (37-45), ISSN 0268-0033

Agrawal, Y., Carey, J., Della, Santina. C., Schubert, M., Minor, L.B. (2010). Diabetes, Vestibular Dysfunction, and Falls: Analyses From the National Health and Nutrition Examination Survey. Otology \& Neurotology, Vol.31,No. 9,(December 2010), pp. (1445-1450), ISSN 1531-7129

Allum, J.H., Budingen, H.J. (1979). Coupled stretch reflexes in ankle muscles: an evaluation of the contributions of active muscle mechanisms to human posture stability. Progress in Brain Research, Vol. 50, No. 1, (n.d. 1979), pp. (185-195), ISSN 0079-6123

Allum, J.H., Honegger, F., Schicks, H. (1993). Vestibular and proprioceptive modulation of postural synergies in normal subjects. Journal of Vestibular Research: Equilibrium $\mathcal{E}$ Orientation, Vol. 3, No. 1, (Spring 1993), pp. (59-85). ISSN 0957-4271

Aniss, A.M., Diener, H.C., Hore, J., Burke, D., Gandevia, S.C. (1990). Reflex activation of muscle spindles in human pretibial muscles during standing. Journal of Neurophysiology, Vol. 64, No. 2, (August 1990), pp. (671-679), ISSN 0022-3077

Aranda-Moreno, C., Meza, A., Rodriguez, R., Mantilla, M.T., Jáuregui-Renaud, K. (2009). Diabetic polyneuropathy may increase the handicap related to vestibular disease. Archives of Medical Research, Vol 40, No. 3, (April 2009), pp. (180-185), ISSN 01884409

Berg, K.O., Wood-Dauphinee, S.L., Williams, J.I., Maki, B.E. (1992). Measuring balance in the elderly: validation of an instrument. Canadian Journal of Public Health, Vol. 83, Suppl 2, (July-August 1992), pp. (S7-S11), ISSN 0008-4263

Bloem, B.R., Allum, J.H., Carpenter, M.G., Honegger, F. (2000). Is lower leg proprioception essential for triggering human automatic postural responses? Experimental Brain Research, Vol. 130, No. 3, (February 2000), pp. (375-391), ISSN 0014-4819

Boucher, P., Teasdale, N., Courtemanche, R., Bard, C., Fleury, M. (1995). Postural stability in diabetic polyneuropathy. Diabetes Care, Vol. 18, No. 5, (May 1995), pp. (638-645), ISSN 0149-5992

Camicioli, R., Howieson, D., Lehman, S. (1997). Talking while walking: the effect of a dual task in ageing and Alzheimer's disease. Neurology, Vol. 48, No. 4 (April 1997), pp. (955-958), ISSN 0028-3878

Cavanagh, P.R., Derr, J.A., Ulbrecht, J.S., Maser, R.E., Orchard, T.J. (1992). Problems with gait and posture in neuropathic patients with insulin- dependent diabetes mellitus. Diabetic Medicine, Vol. 9, No, 5, (June 1992), pp. (469-474), ISSN 0742-3071

Colledge, N.R., Cantley, P., Peaston, I., Brash, H., Lewi,s S., Wilson, J.A. (1994). Aging and balance: the measurement of spontanaeous sway by posturography. Gerontology, Vol. 40, No. 5, (1994), pp. (273-278). ISSN 0734-0664

Courtemanche, R., Teasdale, N., Boucher, P., Fleury, M., Lajoie, Y, Bard C. (1996). Gait problems in diabetic neuropathic patients. Archives of Physical Medicine and Rehabilitation, Vol. 77, No. 9, (September 1996), pp. (849-855), ISSN 0003-9993 
Cruz-Gómez, N.S., Plascencia, G., Villanueva-Padrón, L.A., Jáuregui-Renaud, K. (2011) Influence of obesity and gender on the postural stability during upright stance. Obesity Facts, Vol. 4, No. 3, (June 2011), pp. (212-217), ISSN 1662-4025

De Kleijn, A. Experimental physiology of eth labyrinth. (1923). Journal of Laryngology and Otology, Vol. 38, No. 12, (December 1923), pp. (646-663), ISSN 0022-2151

Delbaere, K., Crombez, G., Vanderstraeten, G., Willems, T., Cambier, D. (2004). Fear-related avoidance of activities, falls and physical frailty. A prospective community-based cohort study. Age and Ageing, Vol. 33, No. 4, (July 2004), pp. (368-373), ISSN 00020729

DeMott, T.K., Richardson, J.K., Thies, S.B., Ashton-Miller, J.A. (2007). Falls and gait characteristics among older persons with peripheral neuropathy. American Journal of Physical Medicine and Rehabilitation, Vol. 86, No. 2, (February 2007), pp. (125-132), ISSN 0894-9115

Diener, H.C., Dichgans, J., Guschlbauer, B., Mau, H.(1984a). The significance of proprioception on postural stabilization as assessed by ischemia. Brain Research, Vol. 296, No. 1, (March 1984), pp. (103-109), ISSN 00068993

Diener, H.C., Dichgans, J., Bootz, F., Bacher, M. (1984b). Early stabilization of human posture after a sudden disturbance: influence of rate and amplitude of displacement. Experimental Brain Research, Vol. 56, No. 1, (August 1984), pp. (126134), ISSN 0014-4819

Diener, H.C., Horak, F.B., Nashner, L.M. (1988). Influence of stimulus parameters on human postural responses. Journal of Neurophysiology, Vol. 59, No. 6, (June 1988), pp. (18881905) ISSN 0022-3077

Dietz, V. Physiology of human gait: neural processes. (2001). Advances in Neurology 2001;Vol. 87, (n.d. 2001), pp. (53-61), ISSN 0091-3952

Dyck, P.J., Karnes, J.L., Daube, J., O'Brien, P., Service, F.J. (1985). Clinical and neuropathological criteria for the diagnosis and staging of diabetic polyneuropathy. Brain, Vol. 108, Pt. 4, (December 1985), pp. (861-880), ISSN 00068950

Era, P., Schroll, M., Ytting, H., Gause-Nilsson, I., Heikkinen, E., Steen, B. (1996). Postural balance and its sensory - motor correlates in 75-year-old men and women: A crossnational comparative study. Journals of Gerontology. Series A, Biological Sciences and Medical Sciences, Vol. 51, No. 2, (March 1996), pp. (M53 -M63), ISSN 1079-5006

Era, P., Sainio, P., Koskinen, S., Haavisto, P., Vaara, M., Aromaa, A. (2006). Postural balance in a random sample of 7,979 subjects aged 30 years and over. Gerontology, Vol. 52, No. 4 (2006), pp. (204-213), ISSN 0304-324X

Fitzpatrick, R.C., Gorman, R.B., Burke, D., Gandevia, S.C. (1992). Postural Proprioceptive Reflexes In Standing Human Subjects: Bandwidth of response and transmission characteristics. Journal of Physiology, Vol. 458, (December 1992), pp. (69-83). ISSN 0022-3751

Forssberg, H., Hirschfeld, H. Postural adjustments in sitting humans following external perturbations: muscle activity and kinematics. (1994). Experimental Brain Research, Vol. 97, No. 3, (January 1994), pp. (515-527), ISSN 0014-4819

Goldberg, A., Russell, J.W., Alexander, N.B. Standing Balance and Trunk Position Sense in Impaired Glucose Tolerance (IGT)-Related Peripheral Neuropathy, (2008). Journal of the Neurological Sciences, Vol. 270, No. 1-2, (July 2008), pp. (165-171), ISSN 0022-510X 
Horak, F.B. (1987). Clinical measurement of postural control in adults. (1987). Physical Therapy, Vol. 67, No. 12, (Dec1987), pp. (1881-1885), ISSN 0031-902

Horak, F.B., Shupert, C.L., Mirka, A. (1989). Components of postural sway dyscontrol in the elderly: a review. Neurobiology of Aging, Vol. 10, No. 6, (November-December 1989), pp. (727-738), ISSN 0197-4580

Horak, F.B., Nashner, L.M., Diener, H.C. (1990). Postural strategies associated with somatosensory and vestibular loss. Experimental Brain Research, Vol. 82, No. 1, (August 1990), pp. (167-177), ISSN 0014-4819

Horak, F.B., Shupert, C.L., Dietz, V., Horstmann, G. (1994). Vestibular and somatosensory contributions to responses to head and body displacements in stance. Experimental Brain Research, Vol 100, No. 1, (July 1994), pp. (93-106), ISSN 0014-4819

Huang, E.S., Karter, A.J., Danielson, K., Warton, E.M., Ahmed, A.T. (2010). The Association Between the Number of Prescription Medications and Incident Falls in a Multiethnic Population of Adult Type-2 Diabetes Patients: The Diabetes and Aging Study. Journal of General Internal Medicine, Vol. 25, No. 2; (February 2010), pp. (141146), ISSN 0884-8734

Inglis, J.T., Horak, F.B., Shupert, Ch.L., Jones-Rycewicz, C. (1994). The importance of somatosensory information in triggering and scaling automatic postural responses in humans. Experimental Brain Research, Vol. 101, No. 1, (September 1994), pp. (159164), ISSN 0014-4819

Jáuregui-Renaud, K., Kovacsovics, B., Vrethem, M., Odjvist L.M., Ledin, T. (1998). Dynamic and Randomized perturbed posturography in the follow-up of patients with polyneuropathy. Archives of Medical Research, Vol. 29, No. 1, (Spring 1998), pp. (3944), ISSN 0188-4409

Jáuregui-Renaud, K., Sánchez, B.M., Ibarra-Olmos, A., González-Barcena, D. (2009). Neurootologic symptoms in patients with type 2 diabetes mellitus. Diabetes Research and Clinical Practice, Vol 84, No. 3, (June 2009), pp. (e45-47). ISSN 0168-8227

Kanade, R.V., Van Deursen, R.W., Harding, K.G., Price, P.E. (2008). Investigation of standing balance in patients with diabetic neuropathy at different stages of foot complications. Clinical Biomechanics (Bristol, Avon), Vol. 23, No. 9 (November 2008), pp. (1183-1191), ISSN 0268-0033

Katoulis, E.C., Ebdon-Parry, M., Lanshammar, H., Vileikyte, L., Kulkarni, J., Boulton, A.J.M. 1997 Gait abnormalities in diabetic neuropathy. Diabetes Care, Vol. 20, No. 12, (December 1997), pp. (1904-1907), ISSN 0149-5992

Kavounoudias, A., Roll, R., Roll, JP. Foot sole and ankle muscle inputs contribute jointly to human erect posture regulation. Journal of Physiology, Vol. 5532, Pt. 3, (May 2001), pp. (869-878), ISSN 0022-3751

Kerr, B., Condon, S.M., McDonald, L.A. (1985). Cognitive spatial processing and the regulation of posture. Journal of Experimental Psychology. Human Perception and Performance, Vol. 11, No. 5, (October 1985), pp. (617-622), ISSN 0096-1523

Kuang, T.M., Tsai, S.Y., Hsu, W.M, Cheng, C.Y., Liu, J.H., Chou, P. (2008). Visual Impairment and Falls in the Elderly: The Shihpai Eye Study. Journal of the Chinese Medical Association, Vol. 71, No. 9, (September 2008) pp. (467-472), ISSN 1726-4901

Lafond, D., Corriveau, H., Prince, F. (2004). Postural control mechanisms during quiet standing in patients with diabetic sensory neuropathy. Diabetes Care, Vol. 27, No. 1, (January 2004), pp. (8173-178), ISSN 0149-5992 
Leigh, J.R., Zee, D.S. (1991). The Neurology of eye movements, F.A. Davis Company, ISBN 0 80365528 2, Philadelphia, United States of America

Lord, S.R., Lloyd, D.G., Li, S.K. (1996). Sensori-motor function, gait patterns and falls in community-dwelling women. Age and Ageing, Vol. 25, No. 4, (July 1996), pp. (292299), ISSN 0002-0729

Lundin-Olsson, L., Nyberg, L., Gustafson, Y. (2000). The mobility interaction fall chart. Physiotherapy Research International, Vol. 5, No. 3, (n.d. 2000), pp. (190-201), ISSN 1358-2267

Magnus, R. (1924). Körperstellung. Springer, Berlin, Germany

Maki, B.E., Holliday, P.J., Topper, A.K. (1991). Fear of falling and postural performance in the elderly. Journal of Gerontology, Vol. 46, No. 4, (July 1991), pp. (M123-131), ISSN 0022-1422

Manor, B., Li, L. (2009). Characteristics of functional gait among people with and without peripheral neuropathy. Gait \& Posture, Vol. 30, No. 2, (August 2009), pp. (253-256), ISSN 0966-6362

Mauritz, K. H., Dietz, V. (1980). Characteristics of postural instability induced by ischaemic blocking of leg afferents. Experimental Brain Research, Vol. 38, No. 1, (January 1980), pp. 117-119, ISSN 0014-4819

Meyer, P.F., Oddsson, L.I., De Luca, C.J. (2004). Reduced plantar sensitivity alters postural responses to lateral perturbations of balance. Experimental Brain Research, Vol 157, No. 4, (August 2004), pp. (526-536), ISSN 0014-4819

Moreland, J.D., Richardson, J.A., Goldsmith, C.H., Clase, C.M. (2004). Muscle weakness and falls in older adults: a systematic review and meta-analysis. Journal of the American Geriatrics Society, Vol. 52, No. 7, (July 2004), pp. (1121-1129), ISSN 00028614

Mueller, M.J., Minor, S.D., Sahrmann, A.S., Schaaf, J.A., Strube, M.J. (1994). Differences in the gait characteristics of patients with diabetes and peripheral neuropathy compared with age-matched controls. Physical Therapy, Vol. 74, No. 4, (April 1994), pp. (299-313), ISSN 0031-9023

Nashner, L.M. (1976). Adapting reflexes controlling the human posture. Experimental Brain Research, Vol. 26, No. 1, (Aug 1976), pp. (59-72), ISSN 0014-4819

Nashner L.M. (1977). Fixed patterns of rapid postural responses among leg muscles during stance. Experimental Brain Research, Vol. 30, No. 1, (October 1977), pp. (13-24), ISSN 0014-4819

Nashner, L.M., Peters, J.F. (1990). Dynamic posturography in the diagnosis and management of dizziness and balance disorders. Neurologic Clinics, Vol. 8, No. 2, (May 1990), pp. (331-349), ISSN 0733-8619

Niino, N., Tsuzuku, S., Ando, F., Shimokata, H. (2000). Frequencies and circumstances of falls in the National Institute for Longevity Sciences, Longitudinal Study of Aging (NILS-LSA). Journal of Epidemiology, Vol. 10, Suppl. 1, (April 2000), pp. (S90 -S94), ISSN 0917-5040

Oliver, D., Britton, M., Seed, P., Martin, F.C., Hopper, A.H. (1997) Development and evaluation of evidence based risk assessment tool (STRATIFY) to predict which elderly inpatients will fall: case-control and cohort studies. British Medical Journal, Vol. 315, No. 7115, pp. (1049-1053), ISSN 0007-1447 
Oliver, D., Daly, F., Martin, F.C., McMurdo, M.E. (2004). Risk factors and risk assessment tools for falls in hospital in-patients: a systematic review. Age and Ageing, Vol. 33, No. 2, (March 2004), pp. (122-130), ISSN 0002-0729

Oppenheim, U., Kohen-Raz, R., Alex, D., Kohen-Raz, A., Azarya, M. (1999). Postural characteristics of diabetic Neuropathy. Diabetes, Vol. 22, No. 2, (February 1999), pp. (328-332), ISSN 0012-1797

Perell, K.L., Nelson, A., Goldman, R.L., Luther, S.L., Prieto-Lewis, N., Rubenstein, L.Z. (2001). Fall risk assessment measures: an analytic review. Journals of Gerontology. Series A, Biological Sciences and Medical Sciences, Vol. 56, No. 12, (December 2001), pp. (M761-766), ISSN 10795006

Petrofsky, J.S., Cuneo, M., Lee, S., Johnson, E., Lohman, E. (2006). Correlation between gait and balance in people with and without Type 2 diabetes in normal and subdued light. Medical Science Monitor, Vol. 12, No. 7, (July 2006), pp. (CR273-281), ISSN 1234-1010

Podsiadlo, D., Richardson, S. (1991). The timed "Up \& Go": a test of basic functional mobility for frail elderly persons. Journal of the American Geriatric Society, Vol. 39, No. 2, (February 1991), pp. (142-148), ISSN 0002-8614

Pollock, A., Durward, B.R., Rowe, P.J., Paul, J.P. (2000). What is balance? Clinical Rehabilitation, Vol. 14, No. 4, (August 2000), pp. (402-406), ISSN 0269-2155

Poulain, I., Giraudet, G. (2008). Age-related changes of visual contribution in posture control. Gait \& Posture Vol. 27, No. 1, (January 2008), pp. (1-7), ISSN 0966-6362

Richardson, J.K., Ching, C., Hurvitz, E.A. (1992) The relationship between electromyographically documented peripheral neuropathy and falls. Journal of the American Geriatric Society, Vol. 40, No. 10, (October 1992), pp. (1008-1112), ISSN 0002-8614

Richardson, J.K., Hurvitz, E.A. (1995). Peripheral Neuropathy: A True Risk Factor for Falls. Journals of Gerontology. Series A, Biological Sciences and Medical Sciences, Vol. 50, No. 4, (July 1995), pp. (M211-215), ISSN 10795006

Richardson, J.K., Thies, S.B., DeMott, T.K., Ashton-Miller, J.A. (2004). A comparison of gait characteristics between older women with and without peripheral neuropathy in standard and challenging environments. Journal of the American Geriatric Society, vol. 52, No. 9, (September 2004), pp. (1532-1537), ISSN ISSN 0002-8614

Rubenstein, L.Z. (2006). Falls in older people: epidemiology, risk factors and strategies for prevention. Age and Ageing, Vol. 35, Suppl 2, (September 2006), pp. (ii37-41), ISSN 0002-0729

Sacco, I.C.N., Amadio, A.C. (2003). Influence of the diabetic neuropathy on the behavior of electromyographic and sensorial responses in treadmill gait. Clinical Biomechanics (Bristol, Avon), Vol. 18, No. 5, (June 2003) pp. (426-434), ISSN 0268-0033

Scott, V., Votova, K., Scanlan, A., Close, J . (2007). Multifactorial and functional mobility assessment tools for fall risk among older adults in community, home-support, long-term and acute care settings. Age and Ageing, Vol. 36, No. 2, (March 2007), pp. 130-139), ISSN 0002-0729

Schwartz, A.V., Vittinghoff, E., Sellmeyer, D.E., Feingold, K.R., de Rekeneire. N., Strotmeyer, E.S., Shorr, R.I., Shinabarger, N.I. (1987). Limited joint motility in adults with diabetes mellitus. Physical Therapy, Vol. 67, No. 2, (February 1987), pp. (215-218), ISSN 0031-9023 
Shumway-Cook, A., Woollacott, M. (2000a). Motor Control: Theory and Practical Applications (2nd edition), MD: Lippincott, Williams and Wilkins, ISBN 1-60831-018-3, Baltimore, Unites States of America

Shumway-Cook, A., Woollacott, M. (2000b). Attentional demands and postural control: the effect of sensory context. Journals of Gerontology. Series A, Biological Sciences and Medical Sciences, Vol 55, No. 1, (January 2000), pp.(M10-16), ISSN 10795006

Simoneau, G.G., Ulbrecht, J.S., Der, J.A., Becker, M.B., Cavanagh, P.R. (1994). Postural Instability in Patients with Diabetic Sensory Neuropathy. Diabetes Care, Vol. 17, No. 12, (December 1994), pp. (1411-1421), ISSN 0149-5992

Talbot, L.A., Musiol, R.J., Witham, E.K., Metter, E. J. (2005). Falls in young, middle-aged and older community dwelling adults: perceived cause, environmental factors and injury. BMC Public Health 2005,5:86 doi:10.1186/1471-2458-5-86, ISSN 1471-2458

Teasdale, N., Simoneau, M. (2001). Attentional demands for postural control: the effects of ageing and sensory reintegration. Gait \& Posture, Vol 14, No. 3, (December 2001), pp. (203-210), ISSN 0966-6362

Thompson, P.D. , Mardsen, C.D. (1996). Clinical Neurological assessment of balance and gait disorders. In: Clinical Disorders of Balance, Posture and Gait. Bronstein A.M., Brandt T., Woollacott M. pp. (79-84), Arnold, ISBN 034060145 0, London, England

Tinetti ME. (1986). Performance-oriented assessment of mobility problems in elderly patients. Journal of the American Geriatric Society, Vol. 34, No. 2, (February 1986), pp. (119-126), ISSN 0002-8614

Tinetti, M.E., Medes de Leon, C.F., Doucette, J.T., Baker, D.I. (1994). Fear of falling and fallrelated efficacy in relationship to functioning among community-living elders. $J$ Gerontology, Vol. 49, No. 3, (May 1994), pp. (M140-147), ISSN 0022-1422

Tinetti, M.E. (2003). Clinical practice. Preventing falls in elderly persons. New England Journal of Medicine, Vol. 348, No. 1, (January 2003), pp. (42-49), ISSN 0028-4793

Toro, B., Nester, C.J., Farren, P.C. (2003). The status of gait assessment among physiotherapists in the United Kingdom. Archives of Physical Medicine and Rehabilitation, Vol. 84, No. 12, (December 2003), pp. (1878-1884), ISSN 0003-9993

Townsend, M.A. (1985). Biped gait stabilization via foot placement. Journal of Biomechanics, Vol. 18, No. 1, (n.d. 1985), pp. (21-38), ISSN 0021-9290

Uccioli, L., Giacomini, P., Monticone, G., Magrin,i A., Durols, A., Bruno, E., Parist, L., Di Girolamo, S., Menzinger. G. (1995). Body sway in diabetic neuropathy. Diabetes Care, Vol 18, No. 3, (March 1995), pp. (339-344), ISSN 0149-5992

Van Deursen, R.W., Simoneau, G.G. (1999). Foot and ankle sensory neuropathy, proprioception, and postural stability. Journal of Orthopedic and Sports Physical Therapy, Vol. 29, No. 12, (December 1999), pp. (718-726), ISSN 0190-6011

Vinik, A.I., Park, T.S., Stansberry, K.B., Pittenger, G.I. (2000). Diabetes neuropathies. Diabetologia, Vol. 43, No. 8, (August 2000), pp. (957-973), ISSN 0012-186X

Vrethem, M., Ledin, T., Ernerudh, J., Odkvist, L.M., Holmgren, H., Moller, C. (1991). Correlation between dynamic posturography, clinical investigation and neurography in patients with polyneuropathy. ORL Journal for Otorhinolaryngology and its Related Specialities, Vol. 53, No. 5, (n.d. 1991) , pp. (294-298), ISSN 0301-1569 
Wang, C.Y., Olson, S.L., Protas, E.J. (2005). Physical-performance tests to evaluate mobility disability in community-dwelling elders. Journal of Aging and Physical Activity, Vol 13, No. 2, (April 2005), pp. (184-197), ISSN 1063-8652

World Health Organization. (2007). A Global report on falls prevention. Epidemiology of falls. World Health Organization, Retrieved from

<http://www.who.int/ageing/publications/Falls_prevention7March.pdf>

Winter, D.A., Scott, S.H. (1991).Technique for interpretation of electromyography for concentric and eccentric contractions in gait. Journal of Electromyography and Kinesiology, Vol. 1, No. 4, (December 1991), pp. (263-269), ISSN 1050-6411

Winter, D.A. (1995). Human balance and posture control during standing and walking Gait $\mathcal{E}$ Posture, Vol 3, No. 4, (December 1995), pp. (193-214), ISSN 0966-6362

Woollacott, M., Shumway-Cook, A. (2002). Attention and the control of posture and gait: a review of an emerging area of research. Gait $\mathcal{E}$ Posture, vol 16, No. 1, (August 2002), pp. (1-14), ISSN 0966-6362 


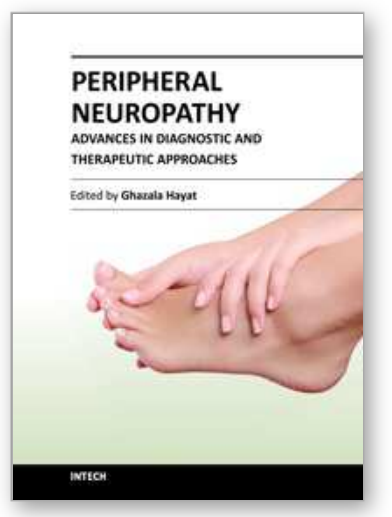

\author{
Peripheral Neuropathy - Advances in Diagnostic and Therapeutic \\ Approaches \\ Edited by Dr. Ghazala Hayat
}

ISBN 978-953-51-0066-9

Hard cover, 206 pages

Publisher InTech

Published online 29, February, 2012

Published in print edition February, 2012

Over the last two decades we have seen extensive progress within the practice of neurology. We have refined our understanding of the etiology and pathogenesis for both peripheral and central nervous system diseases, and developed new therapeutic approaches towards these diseases. Peripheral neuropathy is a common disorder seen by many specialists and can pose a diagnostic dilemma. Many etiologies, including drugs that are used to treat other diseases, can cause peripheral neuropathy. However, the most common cause is Diabetes Mellitus, a disease all physicians encounter. Disability due to peripheral neuropathy can be severe, as the patients suffer from symptoms daily. This book addresses the advances in the diagnosis and therapies of peripheral neuropathy over the last decade. The basics of different peripheral neuropathies is briefly discussed, however, the book focuses on topics that address new approaches to peripheral neuropathies.

\title{
How to reference
}

In order to correctly reference this scholarly work, feel free to copy and paste the following:

Kathrine Jáuregui-Renaud (2012). Polyneuropathy and Balance, Peripheral Neuropathy - Advances in Diagnostic and Therapeutic Approaches, Dr. Ghazala Hayat (Ed.), ISBN: 978-953-51-0066-9, InTech, Available from: http://www.intechopen.com/books/peripheral-neuropathy-advances-in-diagnostic-andtherapeutic-approaches/polyneuropathy-and-balance

\section{INTECH}

open science | open minds

\author{
InTech Europe \\ University Campus STeP Ri \\ Slavka Krautzeka 83/A \\ 51000 Rijeka, Croatia \\ Phone: +385 (51) 770447 \\ Fax: +385 (51) 686166 \\ www.intechopen.com
}

\author{
InTech China \\ Unit 405, Office Block, Hotel Equatorial Shanghai \\ No.65, Yan An Road (West), Shanghai, 200040, China \\ 中国上海市延安西路65号上海国际贵都大饭店办公楼 405 单元 \\ Phone: +86-21-62489820 \\ Fax: $+86-21-62489821$
}


(C) 2012 The Author(s). Licensee IntechOpen. This is an open access article distributed under the terms of the Creative Commons Attribution 3.0 License, which permits unrestricted use, distribution, and reproduction in any medium, provided the original work is properly cited. 